Article

\title{
High Positive MR and Energy Band Structure of $\mathrm{RuSb}_{2+}$
}

\author{
Liang Zhang, Yun Wang and Hong Chang * \\ School of Physical Science and Technology, Inner Mongolia University, Hohhot 010021, China; \\ zl1184078974@aliyun.com (L.Z.); wangyunqw@outlook.com (Y.W.) \\ * Correspondence: changhong@imu.edu.cn
}

Received: 6 June 2020; Accepted: 13 July 2020; Published: 15 July 2020

\begin{abstract}
A high positive magnetoresistance (MR), $78 \%$, is observed at $2 \mathrm{~K}$ on the $a b$ plane of the diamagnetic $\mathrm{RuSb}_{2+}$ semiconductor. On the ac plane, MR is $44 \%$ at $2 \mathrm{~K}$, and about $7 \%$ at $300 \mathrm{~K}$. MR at different temperatures do not follow the Kohler's rule. It suggests that the multiband effect plays a role on the carrier transportation. $\mathrm{RuSb}_{2+}$ is a semiconductor with both positive and negative carriers. The quantum interference effect with the weak localization correction lies behind the high positive MR at low temperature. Judged from the ultraviolet-visible spectra, it has a direct band gap of $1.29 \mathrm{eV}$. The valence band is $0.39 \mathrm{eV}$ below the Fermi energy. The schematic energy band structure is proposed based on experimental results.
\end{abstract}

Keywords: $\mathrm{RuSb}_{2}$; weak localization; magnetoresistivity

\section{Introduction}

Magnetoresistance (MR) is applied in many fields as magnetic memories, magnetic valves, magnetic sensors, and magnetic switches. In spin-polarized materials, MR is observed as the spin-up and spin-down carriers experience different conduction routes. Semiconductors are an important series of materials with many interesting properties [1,2]. MR is also observed in heavily doped n-type nonmagnetic semiconductors, such as $\mathrm{Ge}, \mathrm{Si}, \mathrm{GaAs}$, and CdS [3-6]. In the last decade, MR in organic semiconductors has been one of the hottest research fields [7-10]. In organic semiconductors, the magnetic field generates secondary charge carriers due to dissociation and charge reaction [7-10]. Space charges accumulated at the organic-electrode interfaces change the injection current and account for the tunable MR. Recently, an extremely large MR is observed in Dirac semimetals $\mathrm{Cd}_{3} \mathrm{As}_{2}$ [11], Weyl semimetals $(\mathrm{Mo}, \mathrm{W}) \mathrm{Te}_{2}[12,13]$, and $\mathrm{Bi}_{2} \mathrm{Te}_{3}[14,15]$. It is proposed that $\mathrm{MR}$ in semimetals is due to the increasing severity of the stringency of the hole/electron $=1$ resonance with increasing magnetic field $[12,13]$.

Recently, $\mathrm{AB}_{2}$-type compounds, with $\mathrm{A}$ as heavy transition metals and $\mathrm{B}$ as $\mathrm{Te}, \mathrm{Se}, \mathrm{Sb}$, etc., have attracted a lot of attention due to a series of fascinating properties, such as topological insulating, superconducting, and huge MR [12-14]. In the past, $\mathrm{RuSb}_{2}$ has attracted research attentions with its thermoelectric properties. Different from other thermoelectric compounds, in which the Seebeck coefficient decreases with temperature, $\mathrm{RuSb}_{2}$ has a Seebeck coefficient peak at about $10 \mathrm{~K}$. It is assumed that the huge Seebeck coefficient peak at low temperature is due to its unique band structure $[16,17]$. In theoretical analysis, the energy band gap is formed by the separation between the lifted $d_{x y}$ orbital of the $\mathrm{Ru} / \mathrm{Fe}$ atom and the rest $t_{2 g}$ doublets [18]. $\mathrm{Ru}_{1-x} \mathrm{Mn}_{x} \mathrm{Sb}_{2+\mathrm{d}}$ single crystal has also shown thermoelectric properties [19]. Even though high MR is observed in devices combining a semiconductor and a magnetic material, such as in spin valve transistors [20], magnetic tunnel transistors [21], and stray-field-induced MR devices [22], MR is seldom observed in nonmagnetic single crystals. In nonmagnetic single crystals, MR is closely related to the changed electronic density 
of state by the applied magnetic field. It is able to act as an indicator of some other interesting physical phenomena. In this paper, we studied a high positive MR of $\mathrm{RuSb}_{2+}$ single crystal. Since no experimental work has ever been done on the energy band structure of $\mathrm{RuSb}_{2}$, we also proposed an energy band structure based on the information deduced from the electronic transportation, UV-vis spectra, and XPS valence band.

\section{Experiments}

$\mathrm{RuSb}_{2+}$ single crystal was grown with the self-flux method in a ratio of $\mathrm{Ru}: \mathrm{Sb}=1: 10$. High-purity $\mathrm{Ru}$ and $\mathrm{Sb}$ powder (99.8\%, Alfa Aesar, Haverhill, MA, USA) from Alfa Aesa were mixed together and placed in an evacuated quartz tube. The samples were heated up to $1150^{\circ} \mathrm{C}$ at a rate of $150{ }^{\circ} \mathrm{C} / \mathrm{h}$ and kept at $1150{ }^{\circ} \mathrm{C}$ for $36 \mathrm{~h}$. The samples were cooled down to $700^{\circ} \mathrm{C}$ at a rate of $2{ }^{\circ} \mathrm{C} / \mathrm{h}$, and after that, the extra $\mathrm{Sb}$ flux was decanted in a centrifuge. In our previous paper, $\mathrm{RuSb}_{2+}$ with an extra $\mathrm{Sb}$ was proved by the energy dispersion X-ray spectra (EDX) on a Hitachi S-4500II field-emission scanning electronic microscope (Hitachi, Tokyo, Japan). The phase of the as-grown crystals was characterized using a powder X-ray diffractometer (Bruker D8 Advance, Bruker, Billerica, MA, USA) using Cu $\mathrm{K}_{\mathrm{a}}$ radiation. Single-crystal X-ray diffraction (XRD) was carried out using Bruker Apex II X-ray diffractometer (Bruker Apex II, Billerica, MA, USA) with Mo radiation $\mathrm{K}_{\mathrm{a} 1}(\lambda=0.71073 \AA$ A). Electrical transport and Hall effect between $2 \mathrm{~K}$ and $400 \mathrm{~K}$ were measured on a quantum design physical property measurement system (PPMS, Quantum Design, San Diego, CA, USA). The resistivities were measured using the standard 4-probe technique.The ultraviolet-visible (UV-vis) spectra were obtained using a PerkinElmer Lambda750 UV-vis spectra (PerkinElmer Lambda750, PerkinElmer, Kumamoto, Japan) at room temperature in the wavelength from 200 to $1500 \mathrm{~nm}$ with the sampling pitch of $2 \mathrm{~nm}$. X-ray photoelectron spectroscopy (XPS) was carried out on a Thermo ESCALAB 250Xi with Al Ka photons $(h v=1486.6 \mathrm{eV})$ and a hemispherical energy analyzer (Thermo ESCALAB 250Xi, Thermo, Waltham, MA, USA).

\section{Results and Discussions}

The typical size of the shiny $\mathrm{RuSb}_{2+}$ crystal is about $4 \times 4 \times 4 \mathrm{~mm}^{3}$, as shown in Figure 1a. EDX measurement, as shown in Figure 1b, gives the ratio of $\mathrm{Ru}: \mathrm{Sb}=29.9: 70.1=1: 2.3$. It is close to the composition of $\mathrm{RuSb}_{2}$. The single-crystal XRD pattern, as shown in Figure 1c, is the procession image of the (h k 0) plane of $\mathrm{RuSb}_{2+}$ with the space group Pnnm [16]. In order to confirm the structure, the XRD patterns are measured on the $a b$ and $a c$ planes of the $\mathrm{RuSb}_{2+}$ single crystal, as shown in Figure $1 \mathrm{~d}$. The peaks in the pattern are exactly from $\mathrm{RuSb}_{2+}$ with the space group Pnnm. The lattice constants are $a=0.5951(2) \mathrm{nm}, b=0.6674(1) \mathrm{nm}$, and $c=0.3179$ (1) $\mathrm{nm}$.

Figure 2a shows the temperature dependence of the resistivity measured on the $a b$ and $a c$ planes with the magnetic field out of the plane, $\rho_{a b}$ and $\rho_{a c}$, with crystal size of $1.5 \times 1.5 \times 1.7 \mathrm{~mm}^{3}$. Below the temperature of about $12 \mathrm{~K}$ at $0 \mathrm{~T}$, which is named as $T_{\mathrm{f}}, \rho_{a b}$ has a very slow upturn. In fact, it is almost flat at $0 \mathrm{~T}$. At $7 \mathrm{~T}$, the slow upturning resistivity remains. At $14 \mathrm{~T}, \rho_{a b}$ sharply increases at low temperature. A similar robust flat resistivity is also observed in some semimetals $[11,12]$. In a semimetal, the bottom of the conduction band narrowly overlaps with the top of the valence band. At applied magnetic fields, the carrier concentration changes at the Fermi surface. As a result, MR appears. As the temperature is above $T_{\mathrm{f}}, \rho_{a b}$ decreases. 


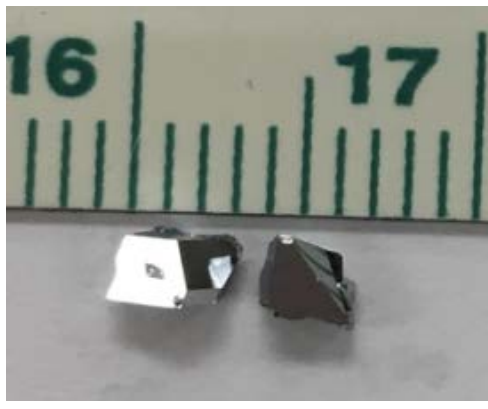

(a)

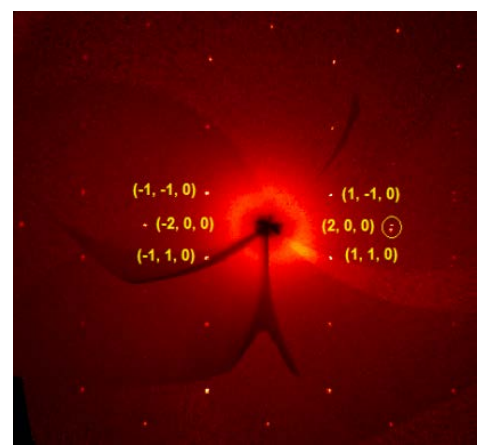

(c)

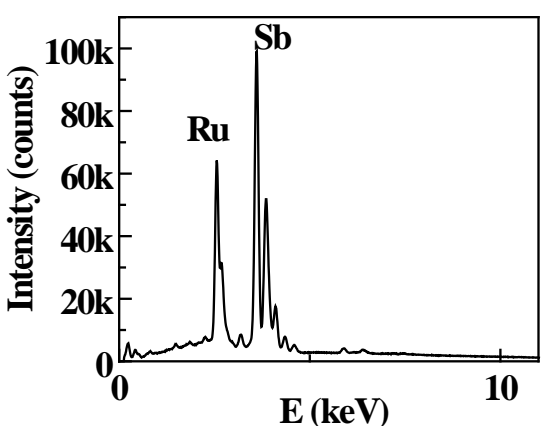

(b)

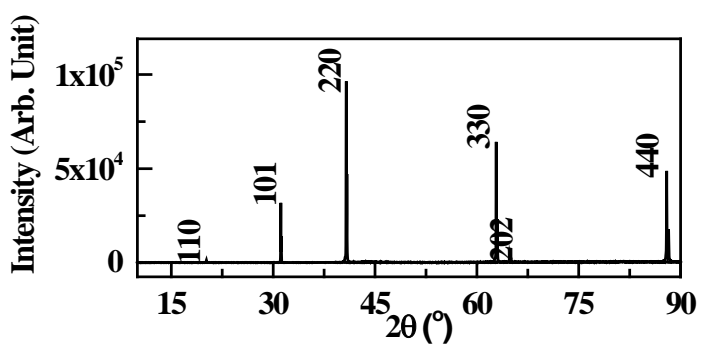

(d)

Figure 1. (a) Image of a single crystal; (b) energy dispersion X-ray (EDX) image; (c) XRD procession image on a single crystal, with the number as the (h k 0) index; (d) powder XRD patterns measured on the $a c$ and $a b$ planes of $\mathrm{RuSb}_{2+}$ single crystal.
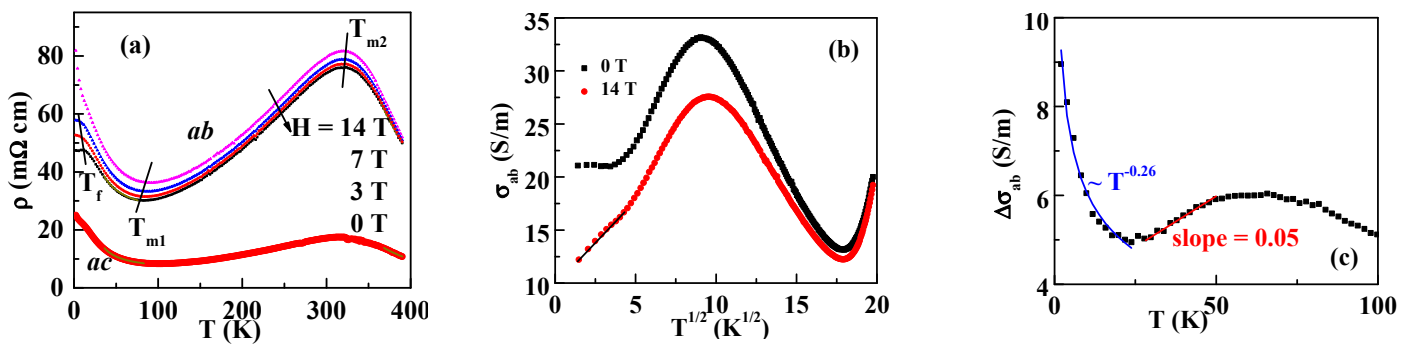

Figure 2. (a) Resistivity versus the temperature of $\mathrm{RuSb}_{2+}$ on the $a c$ plane $\left(\rho_{a c}\right)$ at $0 \mathrm{~T}$ and on the $a b$ plane $\left(\rho_{a b}\right)$ at $0,3,7$, and $14 \mathrm{~T},(\mathbf{b})$ conductivity $\sigma_{a b}$ versus $\sqrt{T}$, and (c) conductivity difference between 0 and $14 \mathrm{~T}$. The solid symbols represent the experimental data, and the solid lines are fitting lines as described in the text.

In a single crystal, the grain boundary contribution is excluded. As the flat (or slow upturning at magnetic field) resistivity at low temperature is related to the semimetal, the quantum interference effects with the weak localization correction are used to explain MR [23]. The total resistivity in the first order is given by

$$
\rho(H, T)=\rho_{0}-\rho_{0}^{2}\left[\sigma_{e e}(H, T)+\sigma_{w l}(H, T)\right]
$$

with $\rho_{0}$ as the residual resistivity, $H$ as the magnetic field, $\sigma_{e e}$ as the conductivity caused by electron-electron interaction effects, and $\sigma_{w l}$ by weak localization, respectively. $\sigma_{e e}$ is expressed as

$$
\sigma_{e e}=\frac{e^{2}}{4 \pi^{2} \hbar} 1.3\left(\frac{4}{3}-\frac{3}{2} F\right) \sqrt{\frac{k_{B} T}{2 \hbar D}}-\frac{e^{2}}{4 \pi^{2} \hbar} F \sqrt{\frac{k_{B} T}{2 \hbar D}} g_{3}(h)
$$


with $h=g \mu_{B} \mu_{0} H /\left(k_{B} T\right)$, the Planck constant $\hbar$, the diffusion constant $D$, and the interaction constant $F . g_{3}(h)$ is a function of $h$ and can be calculated numerically [23]. While the weak localization effect is suppressed by the high magnetic field, the electron-electron interaction is hardly affected [23]. Therefore, the resistivity at $14 \mathrm{~T}$ can be interpreted as the electron-electron interaction. In Equation (2), $\sigma_{e e}$ is proportional to $\sqrt{T}$. As shown in Figure $2 \mathrm{~b}$, below $25 \mathrm{~K}$, the conductivity at $14 \mathrm{~T}$ is linearly fitted with $\sqrt{T}$. It suggests that electron-electron interaction affects the resistivity.

The weak localization contribution at zero field is obtained by subtracting the conductivity at $14 \mathrm{~T}$ from that at zero field. The results are shown in Figure 2c. The $\sigma_{w l}$ term of the weak localization is given by

$$
\begin{aligned}
& \sigma_{w l}=\frac{e^{2}}{2 \pi^{2} \hbar}\left[3 \sqrt{\frac{1}{D \tau_{S O}}+\frac{1}{4 D \tau_{i}}}-\sqrt{\frac{1}{4 D \tau_{i}}}\right]+ \\
& \frac{e^{2}}{2 \pi^{2} \hbar}\left[\frac{e B}{\hbar}\right]^{1 / 2}\left\{f_{3}\left(\frac{B}{B_{2}}\right)+\frac{1}{2 \sqrt{1-\gamma}}\left[f_{3}\left(\frac{B}{B_{+}}\right)-f_{3}\left(\frac{B}{B_{-}}\right)\right]\right\}- \\
& \frac{e^{2}}{\pi^{2} \hbar}\left[\frac{e B_{S O}}{3 \hbar}\right]^{1 / 2}\left[\frac{1}{\sqrt{1-\gamma}}\left(\sqrt{t_{-}}-\sqrt{t_{+}}\right)-\sqrt{t}+\sqrt{t+1}\right]
\end{aligned}
$$

with the inelastic scattering time $\tau_{i}$, the spin-orbit scattering time $\tau_{S O}$, the magnetic induction $B$, the equivalent fields $B_{i}=\frac{\hbar}{4 e D \tau_{i}}, B_{S O}=\frac{\hbar}{4 e D \tau_{S O}}, \gamma=\left(\frac{3 g \mu_{B} B}{8 e D B_{S O}}\right)^{2}, B_{ \pm}=B_{i}+(2 / 3) B_{S O}[1 \pm \sqrt{1-\gamma}]$, $B_{2}=B_{i}+(3 / 4) B_{S O}, t=3 B_{i} /\left(4 B_{S O}\right)$, and $t_{ \pm}=t+(1 / 2)[1 \pm \sqrt{1-\gamma}]$ [23]. The function of $f_{3}$ is defined in Reference [24]. At zero field, $B=0$ in $\mathrm{RuSb}_{2+}$, and $\gamma=0, t_{+}=t+1, t_{-}=t$. Therefore, both the second and third terms are zero. Equation (3) becomes $\sigma_{w l}=\frac{e^{2}}{\hbar}\left[3 \sqrt{\frac{1}{D \tau_{S O}}+\frac{1}{4 D \tau_{i}}}-\sqrt{\frac{1}{4 D \tau_{i}}}\right] \cdot \tau_{i}$ has a temperature dependence as $\tau_{i}=C T^{-p}$, with $p \geq 2$ [23]. As RuSb ${ }_{2+}$ is composed of heavy atoms, $\tau_{S O}$ is expected to be very small and significantly influence $\sigma_{w l}$. It is observed that $\tau_{S O}$ decreases by addition of heavy atoms with increased spin-orbit coupling [25]. Considering that the temperature increases the spin-orbit relaxation time, it is assumed that $\tau_{S O} \sim T^{\delta}$ at low temperature. At low temperature, $\tau_{i}>\tau_{S O}$, so that $\sigma_{w l} \propto T^{-\delta / 2}$ is obtained. As shown in Figure 2c, the conductivity contributed by the weak localization is the difference between 14 and $0 \mathrm{~T}$. Below $25 \mathrm{~K}$, the fitting gives $\sigma_{w l} \sim T^{-0.26}$ with $\delta \approx 0.52$. The low $\delta$ value is consistent with the stable spin-orbit interaction with the temperature. As the temperature increases, $\tau_{i}$ becomes compatible with $\tau_{S O}$, and $\tau_{i}$ cannot be ignored. With a low $\delta$ value, $\tau_{S O}$ is assumed to be a constant at a narrow temperature range. The major temperature factor in $\sigma_{w l}$ is $\tau_{i}$, and $\sigma_{w l} \propto T$ is obtained. As shown in Figure 2c, it is linear between 25 and $50 \mathrm{~K}$. Furthermore, at $T_{\mathrm{m} 2} \sim 312 \mathrm{~K}$, the slope of the resistivity changes from positive to negative, as shown in Figure 2a. It is due to the competing effect of thermally activated and impurity-induced conduction in semiconductors [16], instead of a metallic-insulating transition.

Under the applied magnetic field, the resistivity, measured in either the $a b$ or $a c$ plane, becomes higher in the whole temperature range. MR is defined as $M R=\frac{R(H)-R(0)}{R(0)} \times 100 \%$. MR measured with the current in the $a b$ plane and the applied magnetic field out of the $a b$ plane is shown in Figure $3 a$, and that measured with the current in the $a c$ plane and the applied magnetic field out of the $a c$ plane is shown in Figure $3 b$, and that measured with the current and the applied magnetic field in the $a b$ plane is shown in Figure 3c. Below $T<12 \mathrm{~K}$, as shown above, $\rho_{a b}$ has a slow upturn at $0 \mathrm{~T}$. Both the transversal and longitudinal MR $(a b)$ are very high. It is consistent with the semimetal nature of $\mathrm{RuSb}_{2+}$. In the range of $T_{\mathrm{f}}<T<T_{\mathrm{m} 1}$, both the transversal and the longitudinal MR $(a b)$ decrease quickly but are still higher than that in the range of $T_{\mathrm{m} 1}<T<T_{\mathrm{m} 2}$. While above $T>T_{\mathrm{m} 2}, \mathrm{MR}(a b)$ and MR (ac) decrease very fast.

Figure 3a illustrates the transversal MR $(a b)$ at different temperatures, 2, 10, 20, 35, 50, 100, 200, and $300 \mathrm{~K}$. At $2 \mathrm{~K}$, the positive MR $(a b)$ is as large as $72 \%$ at $14 \mathrm{~T}$. The increasing temperature decreases MR $(a b)$. At $300 \mathrm{~K}$, it is still 4.5\%. Moreover, MR $(a b)$ is not saturated at $14 \mathrm{~T}$. MR $(a c)$, which is measured on the $a c$ plane with the magnetic field out of the plane, is $44 \%$ at $2 \mathrm{~K}$, Figure $3 \mathrm{~b}$. MR $(a c)$ is much smaller than the peer MR $(a b)$ at $2 \mathrm{~K}$. Similar to the resistivity, MR is also anisotropic. Analyzing the origin of the MR, the high longitudinal MR $(a b)$, about $82 \%$ at $2 \mathrm{~K}$, as shown in Figure $3 \mathrm{~b}$, is against the Lorentz force effect at low temperature. The Lorentz force effect may contribute to MR above $50 \mathrm{~K}$ 
in $\mathrm{RuSb}_{2+}$. Generally, the contribution by the Lorentz force is at zero or first order of $k T / E_{\mathrm{f}}$. It is also consistent with the variation of MR above $50 \mathrm{~K}$. At low temperature, the slow upturning $\rho_{a b} \sim T$ plays an important role on the high MR $(a b)$. The MR at low temperature is related to the quantum interference effect, taking the weak localization correction into account. Table 1 lists the MR in other semiconductors with either magnetic or nonmagnetic properties. The transversal MR $(a b)$ in $\mathrm{RuSb}_{2+}$ is higher than that in other semiconductors.
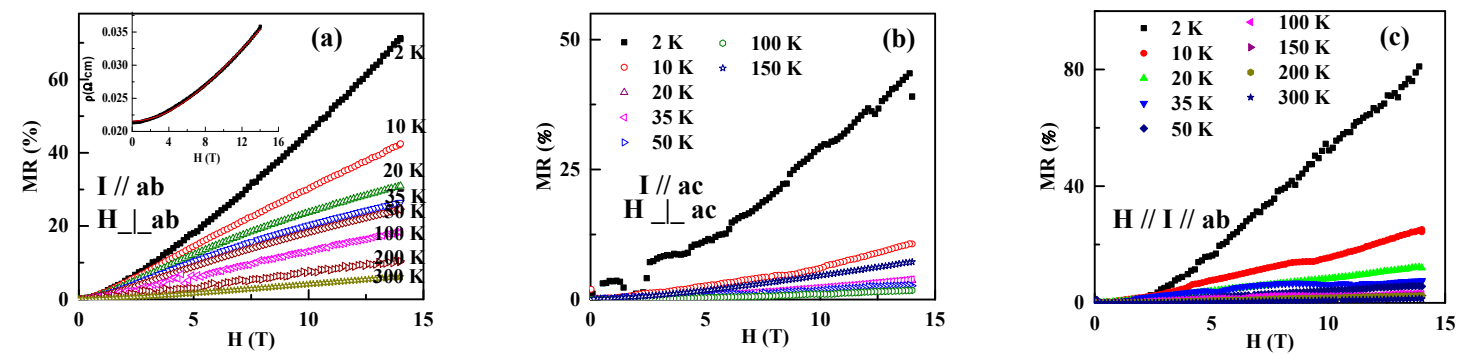

Figure 3. (a) The transversal MR measured with the current $I$ on the $a b$ plane and the magnetic field $H$ out of the $a b$ plane at 2, 10, 20,35,50,100,200, and $300 \mathrm{~K}$; (b) the longitudinal MR with both $I$ and $H$ in the same direction on the $a b$ plane; and (c) the transversal MR measured on the $a c$ plane.

Table 1. Comparison of the MR observed in $\mathrm{RuSb}_{2}$ and other semiconductors.

\begin{tabular}{ccccc}
\hline Compounds & Form & $\begin{array}{c}\text { MR @ Magnetic Field and } \\
\text { Temperature }\end{array}$ & $\begin{array}{c}\text { Magnetic } \\
\text { Properties }\end{array}$ & Ref. \\
\hline $\mathrm{LuPd}_{2} \mathrm{Si}$ & polycrystal & $21 \% @ 8 \mathrm{~T}, 10 \mathrm{~K}$ & Magnetic & {$[26]$} \\
$\mathrm{Tb}_{0.5} \mathrm{Lu}_{0.5} \mathrm{Si}_{3}$ & polycrystal & $60 \% @ 12 \mathrm{~T}, 5 \mathrm{~K}$ & Magnetic & {$[27]$} \\
$\mathrm{Zn}_{0.95} \mathrm{Cu}_{0.05} \mathrm{Cr}_{2} \mathrm{Se}_{4}$ & polycrystal & $>80 \% @ 7 \mathrm{~T}, 3.2 \mathrm{~K}$ & Magnetic & {$[28]$} \\
$\mathrm{CdS}$ & pingle-crystal & $1 \% @ 8 \mathrm{~T}, 1.2 \mathrm{~K}$ & Nonmagnetic & {$[4]$} \\
$\mathrm{GaAs}$ & film & $2 \% @ 0.9 \mathrm{~T}, 50 \mathrm{~K}$ & Nonmagnetic & {$[6]$} \\
$\mathrm{RuSb}_{2+\mathrm{d}}$ & single crystal & $82 \% @ 14 \mathrm{~T}, 2 \mathrm{~K}$ & Nonmagnetic & Present work \\
\hline
\end{tabular}

By investigating the MR, some information about the Fermi surface can be obtained. Kohler's rule describes the scaling law of the MR with temperature. If the MR measured at different temperatures are scalable with the variable $H / \rho_{0}$, the energy band is a single band, and the Fermi surface is symmetric. The scaling of the $\mathrm{RuSb}_{2+}$ single crystal based on Kohler's rule is shown in Figure 4a. Obviously, MR measured at different temperatures do not fall on the same curve. It indicates that the $\mathrm{RuSb}_{2+}$ single crystal does not obey the Kohler's rule. The discrepancy supports that the RuSb $\mathrm{B}_{2+}$ single crystal has a multi-carrier transport. For two-band or multiband materials, the MR is described by the empirical equation as $\rho_{x x}=\frac{A+B H^{2}}{C+D H^{2}}$. The MR of the $\mathrm{RuSb}_{2+}$ single crystal follows this rule very well, as shown in the inset of Figure 3a. Previously, it has been reported that the Hall resistivity of $\mathrm{RuSb}_{2+}$ changes nonlinearly with the magnetic field [19], as shown in Figure 4b. It is consistent with the multiband nature. Furthermore, the Seebeck factor (S) is positive at $300 \mathrm{~K}$, and it decreases with temperature and becomes negative below $60 \mathrm{~K}$, as shown in the inset of Figure 4b [19]. It supports that both positive and negative carriers coexist in $\mathrm{RuSb}_{2+}$. 

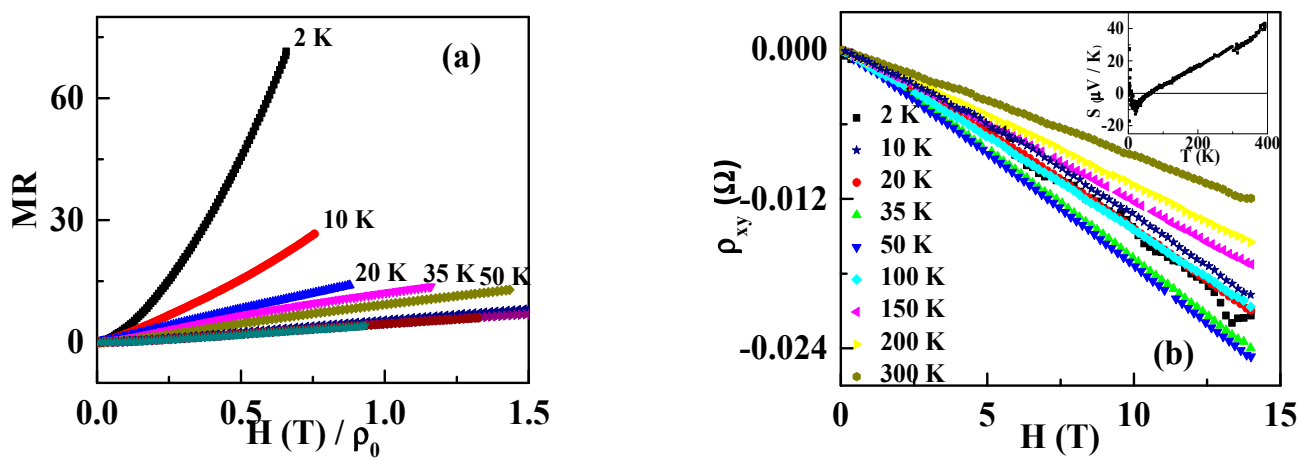

Figure 4. (a) The analysis of MR based on the Kohler's rule; (b) the Hall resistivity at different temperatures, and the inset is the variation of the Seebeck coefficient $S$ with temperature.

In order to study the nature of the band gap of $\mathrm{RuSb}_{2+}$, the UV-vis spectra are measured. Figure $5 \mathrm{a}$ shows $\left[F(R) h v^{2}\right]$ versus $h v . F(R)$ is calculated from the Kubelka-Munk function $F(R)=\left(1-R^{2}\right) / 2 R$, with $R$ as the measured reflection coefficient and $h v$ as the energy of the incident photon [21]. For a direct allowed transition, the band gap energy is the interception at the low-energy side of $\left[F(R) h v^{2}\right]$ versus $h v$. The deduced band gap energy is $1.29 \mathrm{eV}$. An obvious absorption is also observed at $1.29 \mathrm{eV}$, as pointed out by the arrow in the upper inset of Figure 5a. The direct band gap is confirmed by the Tauc relation, which is described as

$$
\alpha h v=K\left(h v-E_{g}\right)^{n}
$$

with $\alpha$ as the absorption coefficient, $K$ as the system-dependent parameter. While $n=1 / 2$, it is direct allowed transition, $n=3 / 2$ for direct forbidden transition, and $n=2$ for indirect allowed transition, and $n=3$ for indirect forbidden transition [21]. The index $n$ is obtained from the logarithmic form of Equation (4),

$$
\ln (\alpha h v)=\ln K+n \ln \left(h v-E_{g}\right)
$$

where $n \approx 0.6$ is derived from the slope of $\ln (\alpha h v) \sim \ln \left(h v-E_{g}\right)$, as shown in the lower inset of Figure $5 \mathrm{a}$. Therefore, $\mathrm{RuSb}_{2+}$ has a direct allowed transition. The deviation from 0.5 is owed to the fractal nature of the density of states due to the disorder in the system [21]. The band gap was reported to be $0.79 \mathrm{eV}$ at $10 \mathrm{~K}$ [20]. The difference between the two results is due to the changed energy band structure by the extra Sb in $\mathrm{RuSb}_{2+}[19]$.
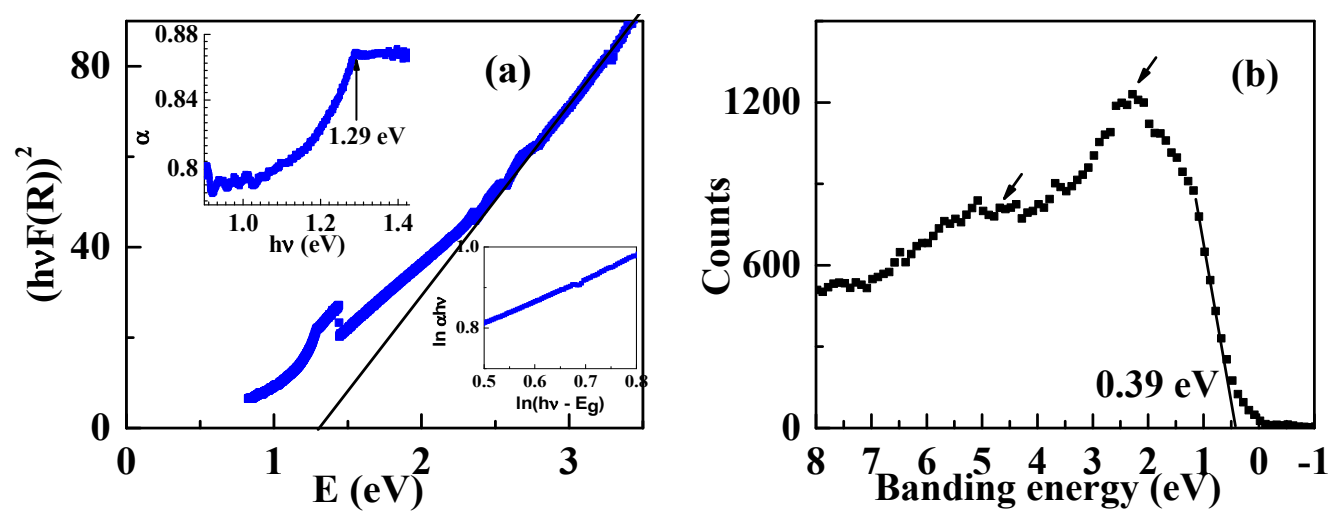

Figure 5. (a) $[h v F(R)]^{2}$ versus the energy $E$, and the upper inset is the corresponding absorption curve, and the lower inset is $\ln (\alpha h v)$ versus $\ln \left(h v-E_{g}\right)$; (b) the valence band of $\mathrm{RuSb}_{2+}$. 
In order to further evaluate the band structure, the XPS valence band spectra are measured and shown in Figure $5 \mathrm{~b}$. The valence band is at $0.39 \mathrm{eV}$ below the Fermi energy. The structures, which are pointed out by the arrows, feature $\mathrm{Ru} 4 d$ electrons [18]. Therefore, the energy band diagram of $\mathrm{RuSb}_{2+}$ is proposed and shown in Figure 6. According to the calculation, the $d$ electrons of the Ru atom have $t_{2 g}$ and $e_{g}$ states, and $t_{2 g}$ is lower than $e_{g}$. The energy band gap is formed by the separation between the $t_{2 g}$ and the valence band.

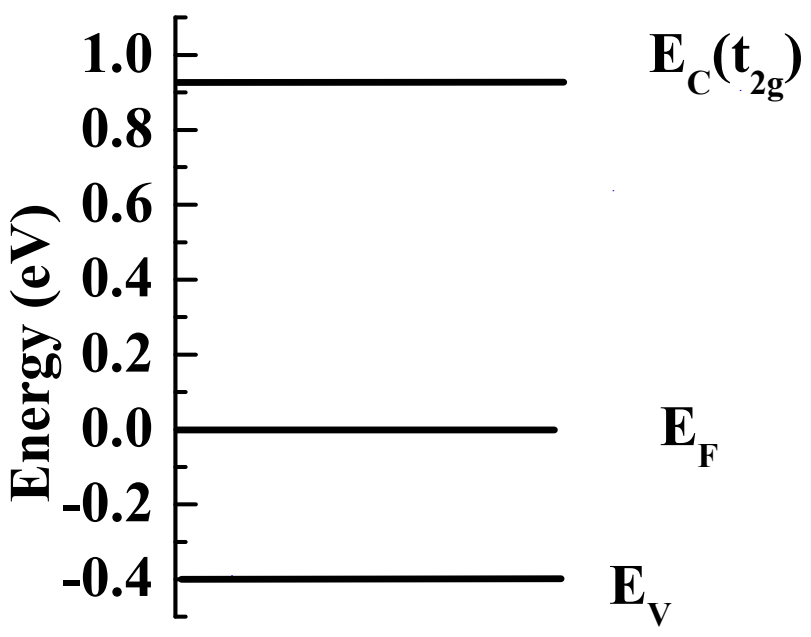

Figure 6. The schematic energy band diagram of $\mathrm{RuSb}_{2+}$.

\section{Conclusions}

A large unsaturated positive MR is observed in single-crystal $\mathrm{RuSb}_{2+}$, especially on the $a b$ plane. A robust slow upturning resistivity in the $\rho_{a b} \sim T$ curve is observed. The MR is smaller on the $a c$ plane without the slow upturning $\rho_{a c} \sim T$. The MR at low temperature is interpreted as the quantum interference effect, taking both the electronic interaction and weak localization correction into account. $\mathrm{RuSb}_{2+}$ has both positive and negative carriers deduced from Hall resistivity and Seebeck coefficient. $\mathrm{RuSb}_{2+}$ is a direct band gap semiconductor, with the band gap as $1.29 \mathrm{eV}$. The valence band lies at $0.39 \mathrm{eV}$ below the Fermi energy. We proposed the schematic energy band diagram of $\mathrm{RuSb}_{2+} \mathrm{based}_{\mathrm{N}}$ the experimental results. Similar robust flat $\rho_{a b} \sim T$ curve and high MR were also observed in $\mathrm{WTe}_{2}$, $\mathrm{MoTe}_{2}, \mathrm{NbSb}_{2}$, etc. $[10,11,25]$. It indicates that $\mathrm{RuSb}_{2+}$ may have similar semimetallic properties as in $\mathrm{WTe}_{2}, \mathrm{MoTe}_{2}, \mathrm{NbSb}_{2}$, etc. However, unlike $\mathrm{WTe}_{2}, \mathrm{RuSb}_{2+}$ is not a topological insulator. In the future, it is worth studying the difference between $\mathrm{WTe}_{2}$ and $\mathrm{RuSb}_{2+}$.

Author Contributions: Conceptualization, L.Z. and H.C.; methodology, H.C.; validation, L.Z., Y.W. and H.C.; formal analysis, L.Z., Y.W. and H.C.; investigation, H.C.; resources, H.C.; data curation, H.C.; writing-original draft preparation, H.C.; writing—review and editing, H.C.; visualization, L.Z., Y.W. and H.C.; supervision, H.C.; project administration, H.C.; funding acquisition, H.C. All authors have read and agreed to the published version of the manuscript.

Funding: This research was funded by the National Natural Science Foundation of China (Grant No. 11864027) and Natural Science Foundation of Inner Mongolia (Grant No. 2020MS01019).

Acknowledgments: The zhong-ke-bai-ce company provided technical support with the XRD measurements.

Conflicts of Interest: The authors declare no conflict of interest.

\section{References}

1. Pinhas, H.; Malka, D.; Danan, Y.; Sinvani, M.; Zalevsky, Z. Design of fiber-integrated tunable thermo-optic C-band filter based on coated silicon slab. J. Eur. Opt. Soc. Rapid Publ. 2017, 13, 32. [CrossRef]

2. Malka, D.; Berke, B.A.; Tischler, Y.; Zalevsky, Z. Improving Raman spectra of pure silicon using super-resolved method. J. Opt. 2019, 21, 075801. [CrossRef] 
3. Fu, J.; Qi, S.; Yang, L.; Dai, Y.; Dai, S. Characterization of Chrysanthemum ClSOC1-1 and ClSOC1-2, homologous genes of SOC1. Plant Mol. Biol. Rep. 2013, 32, 740-749. [CrossRef]

4. Khosla, R.P.; Fischer, J.R. Magnetoresistance in Degenerate CdS: Localized Magnetic Moments. Phys. Rev. B 1970, 2, 4084-4097. [CrossRef]

5. Wan, C.; Zhang, X.; Gao, X.; Wang, J.; Tan, X. Geometrical enhancement of low-field magnetoresistance in silicon. Nature 2011, 477, 304-307. [CrossRef] [PubMed]

6. Sitenko, T.N.; Lyashenko, V.I.; Tyagulskii, I.P. Anisotropy of the magnetoresistance in GaAs monocrystalline films. Phys. Status Sol. 1972, 9, 51-57. [CrossRef]

7. Xiong, Z.; Wu, D.; Vardeny, Z.V.; Shi, J. Giant magnetoresistance in organic spin-valves. Nature 2004, 427, 821-824. [CrossRef]

8. Francis, T.L.; Mermer, O.; Veeraraghavan, G.; Wohlgenannt, M. Large magnetoresistance at room temperature in semiconducting polymer sandwich devices. New J. Phys. 2004, 6, 185. [CrossRef]

9. $\mathrm{Hu}, \mathrm{B} . ; \mathrm{Wu}, \mathrm{Y}$. Tuning magnetoresistance between positive and negative values in organic semiconductors. Nat. Mater. 2007, 6, 985-991. [CrossRef]

10. Santos, T.S.; Lee, J.S.; Migdal, P.; Lekshmi, I.C.; Satpati, B.; Moodera, J.S. Room-temperature tunnel magnetoresistance and spin-polarized tunneling through an organic semiconductor barrier. Phys. Rev. Lett. 2007, 98, 016601. [CrossRef]

11. Liang, T.; Gibson, Q.; Ali, M.N.; Liu, M.; Cava, R.J.; Ong, N.P. Ultrahigh mobility and giant magnetoresistance in the Dirac semimetal $\mathrm{Cd}_{3} \mathrm{As}_{2}$. Nat. Mater. 2014, 14, 280-284. [CrossRef]

12. Ali, M.N.; Xiong, J.; Flynn, S.; Tao, J.; Gibson, Q.D.; Schoop, L.M.; Liang, T.; Haldolaarachchige, N.; Hirschberger, M.; Ong, N.P.; et al. Large, non-saturating magnetoresistance in $\mathrm{WTe}_{2}$. Nature 2014, 514, 205-208. [CrossRef] [PubMed]

13. Zhou, Q.; Rhodes, D.; Zhang, Q.R.; Tang, S.; Schönemann, R.; Balicas, L. Hall effect within the colossal magnetoresistive semimetallic state of $\mathrm{MoTe}_{2}$. Phys. Rev. B 2016, 94, 121101. [CrossRef]

14. Sultana, R.; Neha, P.; Goyal, R.; Patnaik, S.; Awana, V.P.S. Unusual non saturating Giant Magneto-resistance in single crystalline $\mathrm{Bi}_{2} \mathrm{Te}_{3}$ topological insulator. J. Magn. Magn. Mater. 2017, 428, 213-218. [CrossRef]

15. Sultana, R.; Gurjar, G.; Neha, P.; Patnaik, S.; Awana, V.P.S. Hikami-Larkin-Nagaoka (HLN) Treatment of the Magneto-Conductivity of $\mathrm{Bi}_{2} \mathrm{Te}_{3}$ Topological Insulator. J. Supercond. Nov. Magn. 2018, 31, 2287-2290. [CrossRef]

16. Sun, P.; Oeschler, N.; Johnsen, S.; Iversen, B.B.; Steglich, F. Huge Thermoelectric Power Factor: FeSb 2 versus $\mathrm{FeAs}_{2}$ and $\mathrm{RuSb}_{2}$. Appl. Phys. Express 2009, 2, 091102. [CrossRef]

17. Sun, P.; Oeschler, N.; Johnsen, S.; Iversen, B.B.; Steglich, F. Thermoelectric properties of the narrow-gap semiconductors $\mathrm{FeSb}_{2}$ and $\mathrm{RuSb}_{2}$ : A comparative study. J. Phys. Conf. Ser. 2009, 150, 012049. [CrossRef]

18. Gippius, A.; Okhotnikov, K.; Baenitz, M.; Shevelkov, A. Band Structure Calculations and Magnetic Relaxation in Correlated Semiconductors FeSb 2 and $\mathrm{RuSb}_{2}$. Solid State Phenom. 2009, 152, 287-290. [CrossRef]

19. Chang, H.; Gui, X.; Huang, S.; Nepal, R.; Chapai, R.; Xing, L.; Xie, W.; Jin, R. Mn-induced ferromagnetism and enhanced thermoelectric properties in $\mathrm{Ru}_{1}-\mathrm{xMnxSb}_{2+\delta}$. New J. Phys. 2019, 21, 033008. [CrossRef]

20. Anil Kumar, P.S.; Jansen, R.; van't Erve, O.M.J.; Vlutters, R.; Kim, S.D.; Lodder, J.C. 300\% magnetocurrent in a room temperature operating spin-valve transistor. Physics C 2001, 350, 166. [CrossRef]

21. Dijken, S.; Jiang, X.; Parkin, S.S.P. Giant magnetocurrent exceeding 3400\% in magnetic tunnel transistors with spin-valve base layers. Appl. Phys. Lett. 2003, 83, 951-953. [CrossRef]

22. Kubrak, V.; Rahman, F.; Gallagher, B.L.; Main, P.C.; Henini, M.; Marrows, C.H.; Howson, M.A. Magnetoresistance of a two-dimensional electron gas due to a single magnetic barrier and its use for nanomagnetometry. Appl. Phys. Lett. 1999, 74, 2507. [CrossRef]

23. Ziese, M. Searching for quantum interference effects in $\mathrm{La}_{0.7} \mathrm{Ca}_{0.3} \mathrm{MnO}_{3}$ films on $\mathrm{SrTiO}_{3}$. Phys. Rev. B 2003, 68, 132411. [CrossRef]

24. Kawabata, A. Theory of Negative Magnetoresistance I. Application to heavily doped semiconductors. J. Phys. Soc. Jpn. 1980, 49, 628-637. [CrossRef]

25. Reidy, S.G.; Cheng, L.; Bailey, W.E. Dopants for independent control of precessional frequency and dampling in Ni81Fe19 (50 nm) thin films. Appl. Phys. Lett. 2003, 82, 1254. [CrossRef]

26. Rawat, R.; Kushwaha, P.; Das, I. Magnetoresistance studies on $\mathrm{RPd}_{2} \mathrm{Si}(\mathrm{R}=\mathrm{Tb}, \mathrm{Dy}$, Lu) compounds. J. Phys. Condens. Matter 2009, 21, 306003. [CrossRef] 
27. Mukherjee, K.; Iyer, K.K.; Sampathkumaran, E.V. Evolution of a metastable phase with a magnetic phase coexistence phenomenon and its unusual sensitivity to magnetic fifield cycling in the alloys $\mathrm{Tb}_{5}-\mathrm{xLuxSi}_{3}$ (x $\leq$ 0.7). J. Phys. Condens. Matter 2011, 23, 206002. [CrossRef]

28. Parker, D.R.; Green, M.; Bramwell, S.T.; Wills, A.S.; Gardner, J.S.; Neumann, D.A. Crossover from Positive to Negative Magnetoresistance in a Spinel. J. Am. Chem. Soc. 2004, 126, 2710-2711. [CrossRef]

(C) 2020 by the authors. Licensee MDPI, Basel, Switzerland. This article is an open access article distributed under the terms and conditions of the Creative Commons Attribution (CC BY) license (http://creativecommons.org/licenses/by/4.0/). 